\section{Versatile electrical stimulator for providing cardiac-like electrical impulses in vitro}

Stefano Gabetti,1,2 Giovanni Putame, 1,2 Federica Montrone, 1,2 Giuseppe Isu, ${ }^{3}$ Anna Marsano, ${ }^{3}$ Alberto Audenino, 1,2 Diana Massai1,2

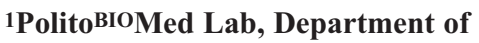
Mechanical and Aerospace Engineering, Politecnico di Torino, Turin, Italy; Interuniversity Center for the Promotion of the 3Rs Principles in Teaching and Research, Italy; 3Department of Surgery and Department of Biomedicine, Basel University Hospital, Basel, Switzerland

\begin{abstract}
In the perspective of reliable methods alternative to in vivo animal testing for cardiac tissue engineering (CTE) research, the versatile electrical stimulator ELETTRA has been developed. ELETTRA delivers controlled and stable cardiac-like electrical impulses, and it can be coupled to already existing bioreactors for providing in vitro combined biomimetic culture conditions. Designed to be cost-effective and easy to use, this device could contribute to the reduction and replacement of in vivo animal experiments in CTE.
\end{abstract}

\section{Introduction}

Cardiac tissue engineering (CTE) aims to develop functional substitutes of native myocardium to be exploited as in vitro models for cardiac development and disease research, and ultimately for cardiac repair. ${ }^{1}$ In the perspective of reliable methods alternative to in vivo animal tests, ${ }^{2}$ bioreactors are technological devices designed to provide biomimetic culture environments in vitro. In CTE they are widely used as model systems to investigate the individual or combined effects of cardiac-like physicochemical stimuli on cardiac cells and substitutes, with the advantage of excluding systemic effects existing in vivo. ${ }^{3}$ Here we present the versatile electrical stimulator ELETTRA, designed to provide controlled cardiac-like electrical impulses for CTE applications and to be integrated in already existing bioreactors.

\section{Materials and Methods}

ELETTRA design was guided by specific requirements: accuracy in mimicking the in vivo pulsatile electric field experienced by human cardiac cells (resting rate $=$ $1.0-1.7 \mathrm{~Hz}$, electric field $=0.1-10.0 \mathrm{~V} / \mathrm{cm}$, pulse duration $=1-2 \mathrm{~ms}) ;{ }^{3}$ versatility to be used with different bioreactors; ease of use; cost-effectiveness. ELETTRA's core consists of an Arduino Due board running a purpose-built software, interfaced to analog and digital peripherals. A user-friendly interface, based on a push button, a rotary
Correspondence: Polito ${ }^{\text {BIOMed Lab, }}$ Department of Mechanical and Aerospace Engineering, Politecnico di Torino, Turin, Italy.

E-mail diana.massai@polito.it

Key words: Electrical stimulation; cardiac tissue engineering; in vitro models; versatility.

Conference presentation: this paper was presented at the Second Centro 3R Annual Meeting - 3Rs in Italian Universities, 2019, June 20-21, University of Genoa, Italy.

Received for publication: 28 October 2019. Accepted for publication: 11 November 2019.

This work is licensed under a Creative Commons Attribution NonCommercial 4.0 License (CC BY-NC 4.0).

CCopyright: the Author(s), 2019

Licensee PAGEPress, Italy

Biomedical Science and Engineering 2019; 3(s3):111 doi:10.4081/bse.2019.111

encoder and an LCD display, allows the tuning of stimulation parameters. Banana sockets are used as output ports and a sensing resistor enables monitoring the current flowing between the electrodes. Control and stimulation subunits allow to separately increase stimulation amplitude and deliver monophasic or biphasic pulses in a wide range (frequency $=0.5-10.0 \mathrm{~Hz}$, amplitude $=0.5-12.0 \mathrm{~V}$, pulse duration $=1-10 \mathrm{~ms}$ ). To test the system, ELETTRA was connected to two carbon rod electrodes (length $=2.5$ $\mathrm{cm}$, diameter $=0.3 \mathrm{~mm}$ ) embedded in a sil-

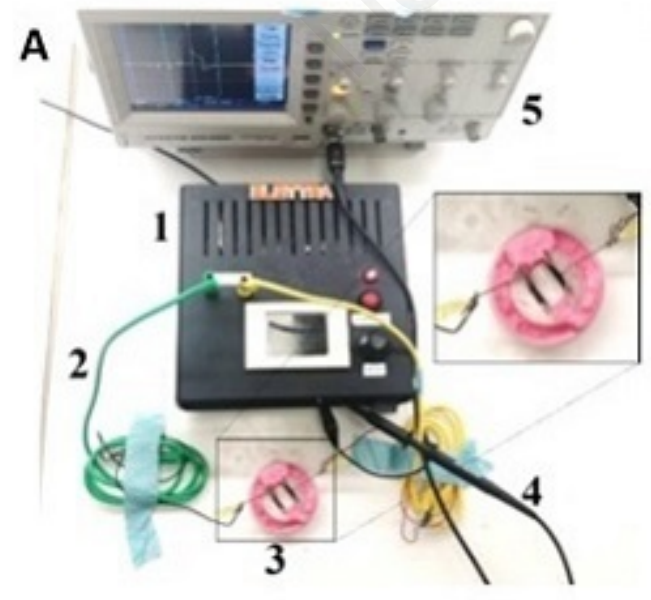

B

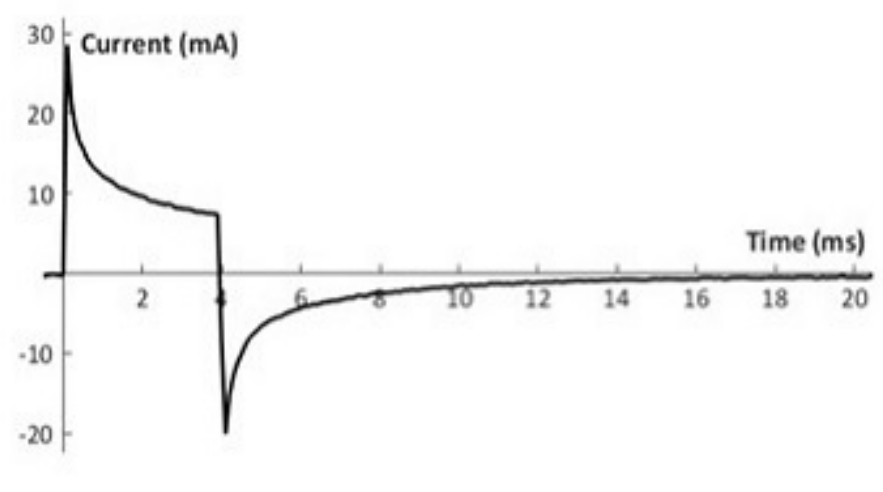

Figure 1. A) Experimental testing setup: 1. ELETTRA; 2. Connectors; 3. Electrode-holder assembly in Petri dish; 4. Sensing circuit probe; 5. Oscilloscope. Inset image: Electrode-holder assembly. B) Plot of the average current vs time for monophasic stimulation (5 V, $1 \mathrm{~Hz}, 4 \mathrm{~ms}$ ) as measured by the sensing circuit. 
icone holder at a fixed distance of $1 \mathrm{~cm}$, and placed in a Petri dish with saline solution (Figure 1A). Monophasic square waves (1 $\mathrm{Hz}, 4 \mathrm{~ms}$ ) with four different amplitudes $(5.0,7.5,10.0,12.0 \mathrm{~V})$ were imposed. A digital oscilloscope was used to measure the total voltage and the voltage drop on the sensing resistor. For each condition, $20 \mathrm{sub-}$ sequent pulses were recorded. Data were processed to calculate the maximum flowing current and the time constant.

\section{Results}

Preliminary tests on ELETTRA confirmed satisfactory stimulation performances, ease of use and cost-effectiveness, with an overall cost below $€ 100$. Voltage amplitude resulted stable during stimulation. As regards the average current between the electrodes (Figure 1B), it increased instantly as the electrical stimulation was provided with a following decrease due to the induced polarization of the solution. During passive phase, the accumulated charges were released in the solution and the current reversed its direction. The maximum current flowing between the electrodes varied from 29 to $79 \mathrm{~mA}$, depending on the imposed voltage. For each condition, the calculated time constant was always lower than $1 \mathrm{~ms}$.

\section{Conclusions}

ELETTRA delivers a controlled and stable stimulation mimicking the cardiac electrical impulses. This device could significantly contribute to the reduction and replacement of in vivo animal experiments for investigation of cardiac development and disease and for preclinical validation of engineered cardiac constructs. Biological tests are ongoing on cardiomyocyte monolayers to investigate the impact of electrical stimulation on cell maturation.

\section{References}

1. Marsano A, Maidhof R, Wan LQ, et al. Scaffold stiffness affects the contractile function of three-dimensional engineered cardiac constructs. Biotechnol Prog 2010;26:1382-90.

2. Russell WMS, Burch RL. The principles of humane experimental technique. London, UK: Methuen; 1959.

3. Massai D, Cerino G, Gallo D, et al. Bioreactors as engineering support to treat cardiac muscle and vascular disease. J Healthc Eng 2013;4:329-70. 